\title{
4 HINFÜHRUNG ZUR UNTERSUCHUNG UND FRAGESTELLUNG
}

Die Literaturanalyse zeigte auf, dass die Thematik um Jugendliche mit türkischem Migrationshintergrund in der Jugendberufshilfe sehr umfangreich ist. Wie bereits festgestellt, ist daher zu prüfen, wie Fachkräfte der Sozialen Arbeit mit dieser Situation umgehen sollten. Weiterhin stellt sich die Frage, ob auf den Islam angesichts seiner zunehmenden Bedeutung für die Jugendlichen in den Maßnahmen der Jugendberufshilfe intensiver eingegangen werden sollte.

Damit Fachkräfte der Sozialen Arbeit auf die beschriebene Situation optimal eingehen können, bedarf es nach Meinung der Verfasserin einer Fortbildung, in der diese Fragen thematisiert werden. Auch Boos-Nünning wies darauf hin, dass eine Qualifizierung des Personals durch Fortbildungen unabdingbar ist.

Die vorliegende Arbeit zeigt auf, welche Aspekte im Umgang mit türkischen Jugendlichen berücksichtigt werden müssen. Aus den resultierenden Ergebnissen werden thematische Inhalte für eine Fortbildung für Fachkräfte der Sozialen Arbeit entwickelt. Aufgrund der Sichtung der Literatur und den daraus resultierenden Überlegungen kristallisiert sich folgende Fragestellung heraus:

\section{Welche Aspekte müssen Fachkräfte der Sozialen Arbeit in der Jugendberufshilfe im Umgang mit türkischen Jugendlichen beachten? \\ - aus der Sicht türkischer Jugendlicher und Fachkräften der Sozialen Arbeit in der Jugendberufshilfe und unter Berücksichtigung des Ansatzes des interreligiösen Dialogs -}

Diese Fragestellung nimmt nicht nur, wie im Titel der Arbeit angedeutet, die Bedeutung des Islam für Jugendliche mit türkischem Migrationshintergrund in Deutschland in den Blick, sondern befasst sich zudem mit weiteren Aspekten, die im Zusammenhang mit der Zielgruppe zu beachten sind. Die Verfasserin hat sich bewusst dazu entschieden die Fragestellung etwas weiter zu formulieren, um eine möglichst genau Betrachtung der Jugendlichen durchzuführen und im Umgang mit Jugendlichen mit türkischem Migrationshintergrund spezifischer arbeiten zu können. 